to pilocarpi 1 when, in fact, the death followed because not enough pilocarpin was used. I was taught to give Norwood's tincture in pneumonia because there was little danger of acute poisoning.' I got over that. Opium will relieve the patient by producing a diaphoresis, yet we do not always give opium similly because we want to render the patient more comfortable. So I believe that aconite in the beginning of pneumonia, where the heart has more than double its burden, is contraindicated because of the ultimate effec of the drug on the heart.

\section{NON-TUBERCULAR HEMORRHAGES OF THE AIR PASSAGES.*}

LAFAYETTE PAGE, A.M., M.D.

Professor of Diseases of the Nose and Throat, Central College of Physicians and Surgeons; Consulting Surgeon on Diseases of the Nose, Throat and Ear, City Hospital and Dispensary. INDIANAPOLIS.

The great vascularity of the mucosa lining the respiratory tract, with its delicate epithelial coverings and its close sympathetic connections through its vasomotor system with the rest of the organism, renders it exceedingly vulnerable to atmospheric conditions and to blood changes and blood pressure from within the organism. The blood vessels supplying this great stretch of mucous membrane are affected by every atmospheric condition, such as thermic and barometric changes, parasitic irritations, dusts and pollens, noxious gases, and all the filth that saturates the atmosphere from many sources. All these forms of irritation disturbing the normal functions of the membrane produce hyperemias, destroy the epithelial coverings and set up morbid processes which weaken the vessel walls and are thus important etiologic factors in the production of hemorrhage. In addition to these atmospheric eauses from without, we have the various forms of traumatism to which the anatomic structure of the respiratory tract is exposed, such as concussions, contusions, fractures, dislocations and the many kinds of surgical procellure for the correction of abnormalities, deformities, removal of growths and mechanical irritation of foreign boties lodged in the respiratory tract. Severe hemorrhages which often result from surgical operations are nsually due to some constitutional diathesis or to some anomaly of the eirculation in the part operated on.

\section{CONSTITUITONAL CAUSES OF HEMORRHAGE.}

On account of the close reflex relationship between the upper respiratory tract and the pelvic organs it has often been observed that any changes taking place in the latter produce vasomotor changes in the nose and throat. The rapid development of the larynx at puberty is always attended by marked hyperemia. The erectile tissue: of the nose show marked engorgement at this period and are especially prone to hemorrhage. Since J. McKenzie, Hack and others have called attention to the close blood relationship of these parts in their physiologic and pathologic changes, we are enabled to treat many cases of hyperemia of the nose and throat with a greater degrea of success.

Fleiss has shown by an extensive series of investigations in the gynecologic clinic of the University of Berlin that there are certain points on the anterior extremity of the middle and inferior turbinate bodies, designated by him the "genital areas," through which influence can be exercised in pathologic conditions of the female pelvic

* Read at the Fifty-fourth Annual Session of the American Med. ical Association. In the Section on Laryngology and Otology, and approved for publication by the Executive Committee : Drs. George organs. By cocainizing the genital areas the pains which accompany or follow the menstrual flow can be relieved, and labor pains can be reduced to a minimum, while by cauterizing these areas a permanent cure of many cases of dysmenorrhea may be achieved.

The writer has had an opportunity of observing in several cases the effect on dysmenorrhea of treatment administered through the genital areas of the turbinates, and also two cases of vicarious hemorrhage, one from the erectile tissues of the nose and the other from the larynx, as the result of sudden arrest of the menstrual flow. There are many interesting cases reported of vicarious hemorrhage from the nose and throat in the literature of the subject. The case of Alibert, in which sudden stoppage of the menstrual flow caused a fatal hemorrhage from the nose, and the case of Kussmaul, quoted by Bosworth, in which there was a periodical nasal hemorrhage in a woman with total absence of uterus; also one by Fricher of a girl of 19 who had never menstruated, but who had violent hemorrhages from the nose at regular intervals of six weeks, which finally proved fatal.

There are a few reports of cases of vicarious hemorrhage due to the arrest of long existing bleeding from rectal hemorrhoids, and from sudden checking of the functions of skin and the cure of certain skin diseases. Bleeding from the nose or larynx during pregnancy is a not uncommon occurrence, probably due to the pressure of the uterus on the large abdominal veins. All of the diseases of the liver, spleen, kidneys, heart and brain which obstruct the return flow of the blood, produce engorgement in the respiratory tract and tend to produce hemorrhage. "We often see persistent hemorrhage from the nose and larynx in cirrhosis of the liver and in renal disease, and it is often associated with the cardiac disease in the venous stases due to failure of compensation in mitral diseases and in aortic insufficiency." (Friedreichs.)

Edgren says that hemorrhages of the air passages are frequent in the presclerotic period and, in the elderly, point to incipient arteriosclerosis. In these cases the bleeding comes on without apparent cause, or it may follow any violent emotion. Any increase of blood pressure is liable to cause rupture of the weakened vessel walls in this disease. The association between the veins of the interior of the skull and the venous sinuses of the nose is so intimate that all the congested states of the cerebrum and meninges are liable to produce epistaxis. Tracheal hemorrhages are comparatively rare. We have an occasional case of fatal hemorrhage from this source due to rupture of an aneurism of the aorta into the trachea. This is due to ulcerations between the rings from pressure of the aneurismal sac. Five cases of tracheal hemorrhage following influenza are reported by Donelan. ${ }^{1}$

\section{IEMORRHAGE DUE TO THE ACUTE FEBRILE DISEASES AND HEMORRHAGIC DIATHESIS.}

Hemorrhages occur as a premonitory symptom or as a concomitant of many of the epidemic fevers, such as typhoid and remittent fever, scurry, diphtheria and the exanthemata. The cause of the hemorrhages in these fevers is yet obscure. They usually occur from the respiratory tract in the initial stages of these affections, and it is the accepted opinion among pathologists that they are due to yet undiscovered changes in the blood and blood vessels of toxic origin.

\footnotetext{
1. Jour. of Laryngol. and Otol., January, 1901.
} 
Niclools claims that in some cases the appearance of hemorrhage corresponds to secondary infections and in others to the cachectic state to which patients are reduced. In the early stages of these affections we find the membrane of the nose and throat intensely hyperemic, and in the later stages a breaking down of the epithelium and vessel walls, with small ulcerations and extravasations of blood.

The hemorrhages of typhoid fever, according to Friedreich, show a predilection for the septum, but may occur in other parts of the mucous membrane. Superficial ulcerations causing hemorrhages are very common in certain regions of the pharynx and larynx. They are commonly found on the faucial pillars, the free border or laryngeal surface of the epiglottis, the aryepiglottic folds, and often occur below the glottis. They are rarely seen on the vocal cords.

Millet observed: "Epistaxis associated with malarial infections is intermittent or periodical, and may be controlled by quinin."

Of all the predisposing causes of hemorrhages hemophilia occupies the first place. It is the "bête noire" of the laryngologist. There are but few of the operators in this department of surgery who have not had sad experiences with this class of patients known as "bleeders." Reports of fatal hemorrhages from excision of the tonsils and adenoids in patients unsuspected of having this predisposition come up before almost every meeting of laryngologists.

The cause of this diathesis is yet undetermined. Statistics show that it has a decided preference to lay its mark on the males. Among the "bleeder" families the females are usually exempt. Among the spontaneous hemorrhages due to hemophilia, epistaxis is by far the most common form. According to one authority, among 236 hemorrhages of various kinds 122 were from the nose, and Eichhorst says: "These hemorrhages are usually preeeded by perversion of the senses of taste and smell. One patient could smell, another taste, the approach of his hemorrhage." Ecchymosis and submucous hemorrhages in the larynx, as well as ulcerations of the pharynx and larynx, are very common in purpura hemorrhagica.

SYPFIMIS AS A CAUSE OF HEMORRTAGES.

Syphilis is not regarded by most of the authorities on the subject as a frequent cause of hemorrhage. In the primary and secondary stages hemorrhages are very rare. In the tertiary and hereditary forms they are much more frequent and are often very severe. It would seem that syphilis would play a most important rôle in the production of hemorrhage from the respiratory tract on account of its predilection for these parts and the destructive changes which are so often wrought here by this disease. It seems that the ulcerative processes rarely invade the blood vessels in syphilis.

\section{IOCAL CAUSES.}

Among the local causes of epistaxis, deflections of the septum should occupy first place. The irregularities on the deformed septum expose the superficial capillaries to many forms of irritants in the air current which denude the vessel walls and thus bring about more or less violent attacks of hemorrhage (Bosworth).

The various forms of neoplasms of the nose, pharynx and larynx, such as fibroma, sarcoma and carcinoma, are often the source of most alarming and difficult hemorrhages to control.
THE MOST FREQUENT LOCATIONS.

"Since the time of Valsalva, a point on the anterior portion of the cartilaginous septum has been known as the most common site of hemorrhage from the nose." Bleeding from this point is rarely dangerous, and is usually easy to control. In hemophilia and purpura the bleeding comes from various points on the mucous membrane. In profuse hemorrhage the bleeding point is difficult, and sometimes impossible, to discover. The ethmoidal veins are often the source of violent hemorrhage, probably on account of their close connection with the cerebral circulation. Other points on the posterior ends of the lower turbinates, the venous plexus in the vault of the pharynx and the venous plexus at the base of the tongue, are frequent seats of hemorrhage. Bleeding from the larynx is comparatively rare, and is not often of a dangerous character from the loss of blood. "Laryngeal hemorrhages are dangerous only when they are beneath the mucous membrane, thus producing hemorrhagic edema and impending asphyxia." They may easily be mistaken for hemorrhage from the lungs or hematemesis.

\section{PROGNOSIS.}

Bleeding from the nose is rarely fatal. In many cases it seems to be Nature's method of relieving overdistended blood vessels. There is no doubt that many cases of cerebral apoplexy are averted by nasal hemorrhage, and it is quite common for some patients to experience immediate relief from a severe congestive headache by a free nose bleed. Spontaneous hemorrhages are more dangerous in young children and the aged on account of the friability of the vessel walls. Fatal hemorrhages of the upper respiratory tract are reported in children under a month old from hereditary syphilis. "Swoboda reports four fatal cases of nurslings from hemorrhages of the upper air passages. In all of his cases the bleeding had not appeared externally, but had passed by the nasopharynx into the stomach." (Sajous' Annual.)

J. N. MeKonzie says: "Persistent and recurrent epistaxis is much more frequent between the ages of 15 and 25 years." The greatest number of fatal hemorrhages come, perhaps, from the removal of the tonsillar and adenoid tissues among those of the hemorrhagic diathesis and those with anomalies of the circulation in these tissues.

DIAGNOSIS.

The importance of a correct diagnosis in hemorrhage of the upper air passages can not be overestimated. Every mechanical means should be exhausted in determining the exact location of the bleeding point. Modern apparatus has been so perfected for diagnostic purposes that no one is justified in assuming to gire an opinion in these cases who has not availed himself of the best apparatus and acquired the best skill possible in their usc. The laryngeal mirror and palate retractor are invaluable aids in locating and differentiating diseases of the whole respiratory apparatus, and in many cases of the digestive apparatus as well.

\section{ILLUSTRATIVE CASES.}

Hemorrhages of the larynx, the nasopharynx, the tonsillar tissues, and the region about the base of the tongue are often mistaken for pulmonary hemorrhage, or hemorrhage of the stomach. The following cases in the writer's experience would tend to emphasize the importance of a patient effort with these instruments (the laryngoscope and palate retractor) in the scarch for the bleeding point. 
CASE 1.-A young attorney of splendid physique called on me for what he supposed were hemorrhages from the lungs. He had been spitting blood for two or three weeks. I could discover no evidence of lung disease by the most careful examination. There was slight hyperemia of the larynx, but no sign of hemorrhage from that source. The anterior ends of the turbinate bodies were considerably engorged, producing occlusion of the nares. The first examination of the post-nasal space, on account of the patient's excessively sensitive reflexes, was unsuccessful. On questioning him he most skilfully evaded any history of syphilis. I remained in darkness as to the source of hemorrhage until I succeeded in examining the postnasal space with the use of the palate retractor. The posterior portion of the septum was completely necrosed, the ulcerated process having extended to the veins in the vault of the pharynx. The hemorrhage that had persisted and was apparently from the lungs soon yielded to syphilitic treatment.

CASE 2.-I obtained the following history from Dr. Graham of Indianapolis:

"I was called to see $\mathrm{Mr}$. E., aged 24, at St. Vincent's Hospital, Oct. 10, 1902. He nad suffered a profuse hemorrhage from the mouth and nose as he was about to alight from a train at the Union Station. I obtained the following history: The patient, so far as he knew, had enjoyed good health until two months previous, when, while in a New York theater, there occurred without any premonition whatever a hemorrhage, the blood flowing from both the nose and mouth. $\mathrm{He}$ was removed to the New York Hospital, and one week later suffered a similar hemorrhage. He stated that it became necessary for the physicians to resort to intravenous injections of salt solution. . . . His case was there diagnosed as one of gastric ulcer, and laparotomy was performed for the same. One week later a third hemorrhage occurred. It was one hour after the fourth hemorrhage occurred that $I$ was called to see this patient. It was stated that this hemorrhage filled a large cuspidor, and I am inclined to believe that this statement is not an exaggeration because of the hemorrhages which I saw at a later period.

"The diagnosis of gastric ulcer was not clear to me in this case and yet I placed my patient at complete rest, withheld all food by the mouth, and placed an ice bag over the epigastric region. . . . Dr. Page was called in consultation, and will give the further history and treatment of this most interesting case. I might add that I wrote Dr. J. H. Lewis, hospital surgeon at the New York Hospital, regarding this case, stating in my letter that I did not think there were any signs or symptoms of gastric ulcer, and received the following letter in reply:

" $\mathrm{Mr}$. E. was a patient at this hospital and was operated on, as he stated to you. His stomach was opened by a twoinch incision in the anterior wall very near the pylorus. A bleeding surface one-half by three-fourths of an inch was found about the middle of the anterior wall and united by mattress sutures of chromated gut. It was thought it had probably been caused by instrumentation, and was not the cause of the previous hemorrhages. Apart from this no ulcer was found. One week after the operation he vomited about one quart of blood. He was discharged in fair condition with the diagnosis still in doubt. One remarkable feature at the operation was profuse and persistent bleeding in the wound through the abdominal wall." "'

I was called in consultation by Dr. Graham to locate, if possible, the seat of hemorrhage in this case. He had suspected that the bleeding was from the throat. Owing to the nervous and exhausted condition of the patient from the great loss of blood, and to the narrowing of the isthmus of the fauces from adhesion of the anterior pillars of the fauces to the dorsum of the tongue, I found it very difficult at the first examination to get a satisfactory view of the larynx and postnasal space. We put the patient on strychnia and after a few days' training I succeeded in getting a good view of the throat in the laryngeal mirror. I discovered at the base of the tongue two deep fissures about an inch and a half long, extending from either side of the epiglottis and cutting the venous plexus about the base of the tongue, coming together and forming an acute angle about an inch above the free border of the epiglottis. The tissue included in this triangle about the root of the tongue was considerably excavated. In examining these fissures with a probe a profuse hemorrhage was started and I had to resort to the suprarenal extract and chromic acid to arrest it. I was satisfied from this examination that this had been the seat of the previous hemorrhages which had so greatly exsanguinated the patient. On discovering these ulcerated fissures and the adhesions between the pillars of the fauces and the tongue (which caused the patient to talk tongue-tied), I concluded that syphilis was the cause of the trouble, although there was absolutely no history to confirm it. I placed the patient on antisyphilitic treatment and applied chromic acid and later nitrate of silver to the ulcerated surfaces. The hemorrhages ceased at once and there was a rapid gain in strength and weight from the start.

CASE 3.- Miss C., aged 23 years, a singer, called on me in June, 1895, for what she supposed were pulmonary hemorrhages. She said the sputum had been examined and that her physician found tubercle bacilli in abundance, and ordered her to Colorado at once. Laryngoscopic examination revealed a small angioma on the left vocal chord surrounded by dried blood. I cleansed the larynx with the spray and could see that the blood vessels about the small growth were dilated and partially denuded of epithelium. She said her hemorrhages usually came on after singing. I requested her to sing, and could detect the blood flowing from the growth and the dilated vessels. I destroyed the distended vessels and the growth with the electro-cautery and there was never any further hemorrhage. The subsequent history of tnis case and the lack of abnormal temperature, as determined by several tests with the thermometer, convinced me that this patient never had tuberculosis, notwithstanding that tubercle bacilli were reported to have been found in the sputum.

\section{TREATMENT.}

It is the intention of the writer to only refer in a few words to some of the numerous methods of treating hemorrhages of the respiratory tract. With our modern method of illuminating the air passages the bleeding point should be located before any efforts at plugging or hemostatics are resorted to for the checking of the hemorrhage. In only exceptional cases where the bleeding is from several different points on the membrane and in those cases where gagging and vomiting render the parts inaccessible should we try to check the flow of blood temporarily until its source can be determined.

The cocain spray and suprarenal extract have proved to be invaluable aids in checking the flow of blood and diminishing the reflexes, thus enabling us to make a diagnosis. The ordinary surgical measures for checking hemorrhages in other parts, such as the use of the ligature and artery forceps, are rarely of service to the laryngologist. Probably in no department of surgery is the operator so handicapped in the treating of hemorrhages as in the nose and throat. Large blood vessels emerge from bony or cartilaginous walls to be distributed to parts accessible only by the most delicate instruments and visible only by artificial lights. To cut down on a vessel and ligate it is out of the question in the majority of cases. In a few cases this has been done successfully in the violent hemorrhage that sometimes follows the removal of the tonsils or adenoids. Keane reports two cases of ligation of the external carotid artery for severe hemorrhage following slight operations in the nose and pharynx. He says this is a simple operation, and every nose and throat surgeon should be prepared to perform it when life is threatened.

For the ordinary hemorrhages of the respiratory tract our resources are ample and effective. Suprarenal or adrenalin extract has proved invaluable in the writer's operative work. 
Some laryngologists claim that it is deceptive in that it is apt to be followed by intense reaction and secondary hemorrhage several hours after an operation.

\section{GENERAL SURGICAL ANESTHESIA. ERNEST J. MELLISH, M.D. \\ Surgeon to Hôtel Dieu (Sisters' Hospital). el paso, TEXas. \\ (Conchided from p. 1.193.)}

\section{SELECTION OF AN ANESTHETIC.}

In order to receive favorable comparative consideration, an anesthetic should possess these qualities: Immediate and remote safety; least disagreeable to take; best calculated to quickly produce and maintain muscular relaxation, followed by the minimum of ill effects, as vomiting, depression, respiratory troubles, renal disturbance; minimum influence on the question of hemorrhage; best adapted to the individual case. In the vast majority of cases the choice lies between ether and chloroform. It has been proven beyond reasonable doubt that, all things considered, chloroform is more dangerous than ether, both immediately and remotely. The immediate safety (during administration) is so overwhelmingly in favor of ether that I do not hesitate to assert that those who hold to the contrary can not be fully acquainted with the facts. There are really very few contraindications to the use of ether when skilfully administered by the "open" method. It is not strongly contraindicated, even in ordinary cases of chronic nephritis, nor in any but acute or severe bronchial affections. In the presence of asthma or of acute bronchial affections, marked arteriosclerosis or atheroma, ether is more dangerous than chloroform, as a rule. Atropin will, to some extent, prevent the danger in the first two conditions, and amyl nitrite or nitroglycerin in the last two. Although formerly (for ten years) a chloroform advocate, I have learned from experience, careful observation, and the study of recent investigations, to use chloroform only where it is especially indicated-and that is extremely seldom. The safety margin between sufficient ether for anesthesia and the lethal dose is much greater than with chloroform. Ether is much safer for indiscriminate use, in that most physicians who administer anesthetics are relatively unskilled in their use. In order to reduce the bad effects of an anesthetic to the minimum, it must be skilfully administered; and when the anesthetic is badly given, the dangers from chloroform are very much greater than those. from ether. Ether kills slowly, generally by its effects on respiration, and usually gives ample warning of danger, since the heart continues to beat long after respiration ceases. Chloroform kills quickly, generally by its effect on circulation, and is very likely to afford no warning of danger. Ether is safer in healthy persons than in the weak and prostrated, while chloroform is often as dangerous in the vigorous as in the debilitated. Treatment usually promptly overcomes unfavorable symptoms from ether, and often does no good in those from chloroform. Public opinion in most parts of this country is overwhelmingly in favor of ether, as compared with chloroform; hence, if an accident happens with ether the surgeon is much more likely to escape blame. As mentioned by Treves, ${ }^{22}$ the objection urged against ether, that it produces venous engorgement, and, consequently, too much oozing from the field of operation, is not well founded, the question being chiefly one of administration.

22. Practitioner, Kondon, October, 1896.
General Statistics. - The statistics of deaths from anesthetics can not be relied on as indicative of the actual dangers encountered. They represent the deaths only, and not the narrow escapes. Moreover, in this country, and in some others, it is probable that not half the deaths from anesthetics are reported.

The statistics presented at the Thirtieth German Surgical Congress, Berlin, May, 1901, credit chloroform with a death rate of one in 2,023 ; ether, one in 5,090 ; ethyl bromid, one in 5,228.

Gurlt's statistics up to 1893 , based on 157,815 war cases, give a death rate for chloroform of one in 2,899 ; chloroform with ether, one in 41,181 ; ethyl bromid, one in 4,588; pental, one in 199. No deaths from ether or A.C.E. mixture reported. ${ }^{23}$

A. I. Bouffleur ${ }^{24}$ quotes statistics of over $1,000,000$ chloroform and over 500,000 ether anesthesias, in which the average chloroform mortality is one in 3,355 , and the average ether mortality one in 16,768.

In considering statistics, we find that the personal equation is of the greatest importance. One man has 15,000 chloroform cases, with only one death, while another has three deaths in less than 1,000 cases. One selects his cases for chloroform, while another gives it indiscriminately. One administers an anesthetic carefully by the open method; another gives it carelessly by the closed or the smothering method. It is notable that by far the greater number of deaths are reported from localities where chloroform and pseudo-scientific, complicated inhalers are extensively employed. I quote from an article in The British Medical Journal of the late '90's: "In a certain institution in Great Britain, in the course of a recent year, there were, out of some 3,000 or 4,000 administrations, no fewer than twelve fatalities from chloroform." Taking the higher number of anesthesias, this means one death in every 333 administrations.

German statistics during the decade 1890 to 1899 , show deaths about as follows:

Ether, one in 7,000; chloroform, one in 2,000; ethyl bromid, one in 4,000 ; pental, one in 2,000 ; chloroform. ether, one in 9,000 ; A.C.E., one in 6,000 .

Ziegler's statistics give the death rate of chloroform as one in 1,900 and of ether as one in 26,000.

Chaput gave it as one in 2,000 and one in 13,000 , respectively.

Foy's statistics, based upon 87\%,507 chloroformizations and 314,738 etherizations, give a mortality of one in 4,301 and one in 14,987, respectively (Sajous' Annual).

Poncet had but one death in 29,000 ether anesthesias. He believes the danger is from asphyxia. ${ }^{25}$

In the presence of postnasal adenoids, especially if hypertrophy of the faucial tonsils also be present, I consider chloroform extremely dangerous. Nitrous oxidwith the patient in the recumbent posture-is the best anesthetic for these cases, and ether, to the stage of primary relaxation, the next best.

Many of the sudden deaths under anesthesia, especially of children and young persons, have occurred in the presence of a persistent, or even hypertrophic, thymus. In most of such cases the spleen and tonsils are unusually large, and enlargement of the follicles at the base of the tongue is constant (L. Laquer, Strassburg).

23. Practical Surgery, Senn, 1901.

24. Chicago Medical Recorder, May, 1897.

25. Progressive Medicine, December, 1902, p. 78 . 